\title{
The influence of mercerised kenaf fibres reinforced polylactic acid composites on dynamic mechanical analysis.
}

\begin{abstract}
The potential of using cellulose to reinforce the thermal stability of kenaf derived cellulose (KDC)/polylactic acid (PLA) composite was investigated in this study. The cellulose was derived from kenaf bast fibre which was chemically treated via chlorination and mercerisation processes. The composites with various loadings of cellulose (dry weight basis) ranging from $0 \%$ to $60 \%$ were produced by melt mixing and compression moulding. Dynamic mechanical properties namely storage modulus (E'), loss modulus (E") and $\tan \delta$ of the KDC/PLA composites and the commercial PLA were analysed and compared as a function of temperature. ESEM micrographs demonstrated that the mercerisation of kenaf fibres have successfully removed the lignin and hemicellulose, thus producing cellulose which can be observed by its rougher surface and greater size reduction than the raw fibre. The DMA results demonstrated that the storage modulus of $60 \% \mathrm{KDC} / \mathrm{PLA}$ composite is twice higher than the commercial PLA and the rest of the composites within a high temperature range (above $80^{\circ} \mathrm{C}$ ). The glass transition temperatures $(\mathrm{Tg})$ generated from the loss modulus curves exhibit that the peak of the loss modulus was shifted to higher temperature as the percentage of the cellulose loading was increased. These results show a better thermal stability of the composites when incorporated with the cellulose.
\end{abstract}

Keyword: Cellulose; DMA; ESEM; Poly(lactic acid) (PLA); Thermal Stability. 